\title{
Inflationary cosmology in unimodular $F(T)$ gravity
}

\author{
Kazuharu Bamba,,$*$ Sergei D. Odintsov, ${ }^{2,3, \dagger}$ and Emmanuel N. Saridakis ${ }^{4,5}$, \\ ${ }^{1}$ Division of Human Support System, Faculty of Symbiotic Systems Science, \\ Fukushima University, Fukushima 960-1296, Japan \\ ${ }^{2}$ Institut de Ciencies de lEspai (IEEC-CSIC), Campus UAB, \\ Carrer de Can Magrans, s/n 08193 Cerdanyola del Valles, Barcelona, Spain \\ ${ }^{3}$ Institució Catalana de Recerca i Estudis Avançats (ICREA), \\ Passeig Lluís Companys, 2308010 Barcelona, Spain \\ ${ }^{4}$ CASPER, Physics Department, Baylor University, Waco, TX 76798-7310, USA \\ ${ }^{5}$ Instituto de Física, Pontificia Universidad de Católica de Valparaíso, Casilla 4950, Valparaíso, Chile
}

\begin{abstract}
We investigate the inflationary realization in the context of unimodular $F(T)$ gravity, which is based on the $F(T)$ modification of teleparallel gravity, in which one imposes the unimodular condition through the use of Lagrange multipliers. We develop the general reconstruction procedure of the $F(T)$ form that can give rise to a given scale-factor evolution, and then we apply it in the inflationary regime. We extract the Hubble slow-roll parameters that allow us to calculate various inflation-related observables, such as the scalar spectral index and its running, the tensor-to-scalar ratio, and the tensor spectral index. Then, we examine the particular cases of de Sitter and powerlaw inflation, of Starobinsky inflation, as well as inflation in a specific model of unimodular $F(T)$ gravity. As we show, in all cases the predictions of our scenarios are in a very good agreement with Planck observational data. Finally, inflation in unimodular $F(T)$ gravity has the additional advantage that it always allows for a graceful exit for specific regions of the model parameters.
\end{abstract}

PACS numbers: $98.80 .-\mathrm{k}, 98.80 . \mathrm{Cq}, 04.50 . \mathrm{Kd}$

\section{INTRODUCTION}

According to the Standard Model of Cosmology, supported by a large amount of observational data, during its evolution the universe has experienced two phases of accelerated expansion, the inflationary one at early times and the current one at late times [1 $[6]$. In order to explain these accelerated phases one should introduce small deviations to the standard physics paradigm, and there are two main directions that one can follow. The first is to maintain general relativity as the gravitational theory and alter the content of the universe by introducing novel, exotic forms of matter fields, either as inflaton field(s) [7] or as dark energy sector [8, 9]. The second way is to modify the gravitational sector, constructing a theory that possesses general relativity as a particular limit, but with additional degrees of freedom that can drive an accelerating expansion [10].

Although most of the works in modified gravity start from the usual gravitational description based on curvature and modify the Einstein-Hilbert action, with the simplest example being the $F(R)$ scenario [11], one could equally well construct gravitational modifications starting from the torsion-based description of gravity. In particular, one could start from the Teleparallel Equivalent of General Relativity (TEGR) 12 15], in which the gravitational Lagrangian is the torsion scalar $T$, and build various extensions, with the simplest example being the

\footnotetext{
*Electronic address: bamba@sss.fukushima-u.ac.jp

${ }^{\dagger}$ Electronic address: odintsov@ieec.uab.es

‡Electronic address: Emmanuel_Saridakis@baylor.edu
}

$F(T)$ theory [16, 17] (for a review see [18]). Interestingly enough, although TEGR is completely equivalent with general relativity at the level of equations, $F(T)$ does not coincide with $F(R)$ gravity, and thus its cosmological application has led to novel features, either at inflationary stage [19] or at the late-time accelerated epoch 20, 21].

On the other hand, unimodular gravity 22] is an interesting gravitational theory, which can be considered as a specific case of general relativity. In particular, while in standard general relativity the origin of the cosmological constant is not well understood 23], in unimodular gravity it arises as the trace-free part of the gravitational field equations, as long as the determinant of the metric is fixed to a number or a function. The great theoretical advantage of this procedure is that since the tracefree part of the field equations is not related to the vacuum expectation value of any matter field, one can fix its value without facing the cosmological constant problem. Hence, one can use unimodular gravity in order to describe the inflationary regime [24] and the late-time cosmic acceleration [25]. Additionally, one can start from unimodular gravity in order to construct extensions, such as unimodular $F(R)$ gravity [26] and unimodular $F(T)$ gravity [27], which prove to have interesting cosmological implications.

In the present work we are interesting in investigating inflationary cosmology in the framework of unimodular $F(T)$ gravity. Specifically, we study the Lagrange multiplier method to represent the action of unimodular $F(T)$ gravity and we build its reconstruction procedure. In addition, we extract the observables of the inflationary regime, namely the spectral index of the curvature perturbations and its running, the tensor spectral index, 
and the tensor-to-scalar ratio, showing that they are in very satisfactory agreement with observations. Finally, we study the instability of the de Sitter solution, by investigating its perturbations, showing that a graceful exit can always be realized for specific parametric regions, which is an advantage of inflation in unimodular $F(T)$ gravity.

The plan of the work is the following. In Sec. II we formulate unimodular $F(T)$ gravity using Lagrange multipliers and we present the reconstruction procedure. In Sec. III we investigate the inflationary realization in the context of unimodular $F(T)$ gravity, in the case of de Sitter, power-law and Starobinsky inflation, as well as for a specific $F(T)$ form, extracting the inflationary observables and comparing them with the Planck data. In Sec. IV we discuss the graceful exit from inflation in the scenario at hand. Finally, Sec. $\mathrm{V}$ is devoted to the conclusions.

\section{UNIMODULAR $F(T)$ GRAVITY}

In this section we will present unimodular $F(T)$ gravity and then we will formulate it using Lagrange multipliers. Finally, we will provide the method for reconstructing unimodular $F(T)$ gravity in the general case.

\section{A. Teleparallel and $F(T)$ gravity}

In teleparallel formulation of gravitation one uses the vierbeins $e_{A}^{\mu}$ as dynamical variables, which at each point $x^{\mu}$ of a generic manifold form an orthonormal base for the tangent space. The metric is then given as

$$
g_{\mu \nu}=\eta_{A B} e_{\mu}^{A} e_{\nu}^{B}
$$

where greek indice span the coordinate space while latin indices span the tangent space). Furthermore, one uses the curvatureless Weitzenböck connection [14] $\stackrel{\mathrm{w}}{\Gamma}_{\nu \mu}^{\lambda} \equiv$ $e_{A}^{\lambda} \partial_{\mu} e_{\nu}^{A}$, instead of the standard torsionless Levi-Civita one, and thus the gravitational field is described not by the curvature tensor but by the torsion one, which reads as

$$
T_{\mu \nu}^{\rho} \equiv e_{A}^{\rho}\left(\partial_{\mu} e_{\nu}^{A}-\partial_{\nu} e_{\mu}^{A}\right)
$$

The Lagrangian of such a theory is just the torsion scalar $T$, which is constructed by contractions of the torsion tensor, namely [15]

$$
T \equiv \frac{1}{4} T^{\rho \mu \nu} T_{\rho \mu \nu}+\frac{1}{2} T^{\rho \mu \nu} T_{\nu \mu \rho}-T_{\rho \mu}{ }^{\rho} T_{\nu}^{\nu \mu} .
$$

Since in $F(R)$ gravity one extends the Einstein-Hilbert Lagrangian, namely the Ricci scalar $R$ to an arbitrary function $F(R)$, one can follow a similar procedure in the context of teleparallel gravity, i.e. generalize $T$ to $F(T)$ obtaining the $F(T)$ gravitational modification [16 18]:

$$
S=\int d^{4} x e\left[\frac{F(T)}{2 \kappa^{2}}\right],
$$

where $e=\operatorname{det}\left(e_{\mu}^{A}\right)=\sqrt{-g}$ and $\kappa^{2}=8 \pi G=M_{p}^{-1}$ is the gravitational constant, with $M_{p}$ the Planck mass.

The equations of motion for $F(T)$ gravity arise by variation of the total action $S+S_{\mathrm{M}}$, where $S_{\mathrm{M}}$ is the matter action, in terms of the vierbeins, and they write as

$$
\begin{aligned}
& e^{-1} \partial_{\mu}\left(e e_{A}^{\rho} S_{\rho}{ }^{\mu \nu}\right) \frac{d F(T)}{d T}+e_{A}^{\rho} S_{\rho}^{\mu \nu} \partial_{\mu}(T) \frac{d^{2} F(T)}{d T^{2}} \\
& -\frac{d F(T)}{d T} e_{A}^{\lambda} T^{\rho}{ }_{\mu \lambda} S_{\rho}{ }^{\nu \mu}+\frac{1}{4} e_{A}^{\nu} F(T)=\frac{\kappa^{2}}{2} e_{A}^{\rho} T^{(\mathrm{M})}{ }_{\rho}^{\nu},(5)
\end{aligned}
$$

where the "super-potential" tensor $S_{\rho}{ }^{\mu \nu}=$ $\frac{1}{2}\left(K_{\rho}^{\mu \nu}+\delta_{\rho}^{\mu} T_{\alpha}^{\alpha \nu}-\delta_{\rho}^{\nu} T_{\alpha \mu}^{\alpha \mu}\right)$ is defined in terms of the co-torsion tensor $K_{\rho}^{\mu \nu}=-\frac{1}{2}\left(T_{\rho}^{\mu \nu}-T_{\rho}^{\nu \mu}-T_{\rho}^{\mu \nu}\right)$. Additionally, $T^{(\mathrm{M})}{ }_{\rho}^{\nu}$ denotes the energy-momentum tensor corresponding to $S_{\mathrm{M}}$. We mention that in the case where $F(T)=T$ one obtains teleparallel equivalent of general relativity, in which case equations (5) coincide with the field equations of the latter.

\section{B. Unimodular conditions}

Let us now present briefly the basic idea of unimodular gravity. In such a construction the determinant $g$ of the metric $g^{\mu \nu}$ is imposed to be a constant value, namely the metric components are constrained in order for $\sqrt{-g}$ to be fixed. Without loss of generality, one can set $\sqrt{-g}=1$ [26]. In the case of cosmological applications one considers a flat Friedmann-LemaîtreRobertson-Walker (FLRW) space-time with metric

$$
d s^{2}=d t^{2}-a^{2}(t)\left[\left(d x^{1}\right)^{2}+\left(d x^{2}\right)^{2}+\left(d x^{3}\right)^{2}\right],
$$

which arises from the vierbein

$$
e_{\mu}^{A}=\operatorname{diag}(1, a(t), a(t), a(t)),
$$

where $a(t)$ is the scale factor and $t$ is the cosmic time. Introducing a new time variable $\tau$ through

$$
d \tau \equiv a^{3}(t) d t
$$

the FLRW metric is rewritten as

$$
d s^{2}=a^{-6}(\tau) d \tau^{2}-a^{2}(\tau)\left[\left(d x^{1}\right)^{2}+\left(d x^{2}\right)^{2}+\left(d x^{3}\right)^{2}\right]
$$

with $a(\tau) \equiv a(t(\tau))$. In this case we have $g_{\mu \nu}=$ $\operatorname{diag}\left(a^{-6}(\tau),-a^{2}(\tau),-a^{2}(\tau),-a^{2}(\tau)\right)$ and the vierbein components are given by

$$
e_{\mu}^{A}=\operatorname{diag}\left(a^{-3}(\tau), a(\tau), a(\tau), a(\tau)\right) .
$$

Hence, we can easily verify the satisfaction of the unimodular gravity constraint, namely that $|e|=\operatorname{det}\left(e_{\mu}^{A}\right)=$ $\sqrt{-g}=1$. 


\section{Lagrange multiplier formulation of unimodular $F(T)$ gravity}

In this subsection we will present the Lagrange multiplier formulation of unimodular $F(T)$ gravity, following the corresponding procedure developed for $F(R)$ gravity in [26]. In particular, we will use the Lagrange multiplier method [28] in the framework of $F(T)$ gravity in order to ensure that the unimodular condition is satisfied. Introducing the Lagrange multiplier $\lambda$, the action of unimodular $F(T)$ gravity with matter can be written as 27]

$$
S=\int d^{4} x\left\{|e|\left[\frac{F(T)}{2 \kappa^{2}}-\lambda\right]+\lambda\right\}+S_{\mathrm{M}},
$$

and in the following we set $2 \kappa^{2}=1$ for simplicity.

By varying the action in Eq. (11) with respect to the vierbein we acquire [27]

$$
\begin{aligned}
& e^{-1} \partial_{\mu}\left(e e_{A}^{\rho} S_{\rho}{ }^{\mu \nu}\right) \frac{d F(T)}{d T}+e_{A}^{\rho} S_{\rho}{ }^{\mu \nu} \partial_{\mu}(T) \frac{d^{2} F(T)}{d T^{2}} \\
& -\frac{d F(T)}{d T} e_{A}^{\lambda} T^{\rho}{ }_{\mu \lambda} S_{\rho}{ }^{\nu \mu}+\frac{1}{4} e_{A}^{\nu}[F(T)-\lambda]=\frac{1}{4} e_{A}^{\rho} T_{\rho}^{(\mathrm{M})}{ }_{\rho}^{\nu} .
\end{aligned}
$$

In the following we consider the matter energymomentum tensor $T^{(\mathrm{M})}{ }_{\rho}^{\nu}$ to correspond to a perfect fluid, namely $T^{(\mathrm{M})}{ }_{\rho}{ }^{\nu}=\operatorname{diag}\left(\rho_{\mathrm{M}},-P_{\mathrm{M}},-P_{\mathrm{M}},-P_{\mathrm{M}}\right)$, where $\rho_{\mathrm{M}}$ and $P_{\mathrm{M}}$ are the energy density and pressure respectively.

In the case of FRLW geometry of (7) or (10), the torsion scalar $T$ defined in (3) becomes

$$
T=-6 H(t)^{2}=-6 a^{6}(\tau) \mathcal{H}(\tau)^{2},
$$

where $\mathcal{H}(\tau) \equiv \frac{1}{a(\tau)} \frac{d a(\tau)}{d \tau}$ is the new function that plays the role of the Hubble parameter $H(t) \equiv \dot{a}(t) / a(t)$ (with dots denoting derivatives with respect to $t$ ). Hence, in this case the general field equations (12) give rise to the two Friedmann equations as

$$
\begin{gathered}
12 a^{6}(\tau) \mathcal{H}^{2} \frac{d F(T)}{d T}+[F(T)-\lambda]-\rho_{\mathrm{M}}=0 \\
-48 a^{12}(\tau) \mathcal{H}^{2}\left(3 \mathcal{H}^{2}+\frac{d \mathcal{H}}{d \tau}\right) \frac{d^{2} F(T)}{d T^{2}}+[F(T)-\lambda] \\
+4 a^{6}(\tau)\left(6 \mathcal{H}^{2}+\frac{d \mathcal{H}}{d \tau}\right) \frac{d F(T)}{d T}+P_{\mathrm{M}}=0 .
\end{gathered}
$$

Eliminating the term $(F(T)-\lambda)$ between (14) and (15), and using the relation (13) we acquire

$4 a^{6}(\tau)\left(3 \mathcal{H}^{2}+\frac{d \mathcal{H}}{d \tau}\right)\left[2 T \frac{d^{2} F(T)}{d T^{2}}+\frac{d F(T)}{d T}\right]+\rho_{\mathrm{M}}+P_{\mathrm{M}}=0$.

Finally, the system of equations closes by considering the continuity equation for the matter fluid, namely

$$
\frac{d \rho_{\mathrm{M}}}{d \tau}+3 \mathcal{H}\left(\rho_{\mathrm{M}}+P_{\mathrm{M}}\right)=0
$$

\section{Reconstruction of unimodular $F(T)$ gravity}

In this subsection we will present the method of reconstructing the $F(T)$ form that generates a given scalefactor evolution. We start by differentiating (13) in order to obtain the useful relation

$$
\frac{d T}{d \tau}=-12 a^{6}(\tau) \mathcal{H}\left(3 \mathcal{H}^{2}+d \mathcal{H} / d \tau\right)
$$

and inserting it into Eq. (16) we acquire

$$
-\frac{1}{3 \mathcal{H}}\left[2 T \frac{d}{d \tau}\left(\frac{d F(T)}{d T}\right)+\frac{d F(T)}{d \tau}\right]+\rho_{\mathrm{M}}+P_{\mathrm{M}}=0 .
$$

Let us now consider a specific scale factor. For simplicity we choose the general power-law form

$$
a(t)=a_{*}\left(\frac{t}{t_{*}}\right)^{p}
$$

where $a_{*}$ is a value of $a$ at time $t_{*}$ and $p$ is a constant, but the reconstruction procedure can be applied in a general $a(t)$ too. In this case relation (8) leads to

$$
\tau=\frac{a_{*}^{3} t_{*}}{3 p+1}\left(\frac{t}{t_{*}}\right)^{3 p+1}
$$

and in terms of the new time variable $\tau$ the above scale factor reads as

$$
a(\tau)=\left(\frac{\tau}{\tau_{*}}\right)^{q}
$$

with

$$
\begin{aligned}
q & \equiv \frac{p}{3 p+1}, \\
\tau_{*} & \equiv \frac{t_{*}}{a_{*}^{1 / p}(3 p+1)} .
\end{aligned}
$$

In this case $H=p / t$ and $\mathcal{H}=q / \tau$, and hence (13) leads to

$$
T=-6\left(\frac{q}{\tau_{*}}\right)^{2}\left(\frac{\tau}{\tau_{*}}\right)^{2(3 q-1)} .
$$

Thus, we can easily find that $d / d T=$ $-\left\{\tau_{*}^{3} /\left[12 q^{2}(3 q-1)\right]\right\}\left(\tau / \tau_{*}\right)^{-3(2 q-1)} d / d \tau . \quad$ Additionally, if the matter perfect fluid has a constant equation-of-state parameter $w=P_{\mathrm{M}} / \rho_{\mathrm{M}}$, then the continuity equation (17) gives $\rho_{\mathrm{M}}=\rho_{\mathrm{M} *}\left(\tau / \tau_{*}\right)^{-3 q(1+w)}$, where $\rho_{\mathrm{M} *}$ is the value of $\rho_{\mathrm{M}}$ at $\tau=\tau_{*}$. Inserting these into Eq. (19) we obtain

$$
\begin{aligned}
\frac{d^{2} F(\tau)}{d \tau^{2}} & +\frac{(2-3 q)}{\tau} \frac{d F(\tau)}{d \tau} \\
& -\frac{3 q(3 q-1)(1+w) \rho_{\mathrm{M} *}}{\tau_{*}^{-3 q(1+w)}} \tau^{-3 q(1+w)-2}=0,
\end{aligned}
$$


which is a differential equation in terms of $\tau$. The general solution of (25) reads as

$$
F(\tau)=c_{1} \tau^{3 q-1}-\frac{(3 q-1) \rho_{\mathrm{M} *}}{[3 q(2+w)-1]}\left(\frac{\tau}{\tau_{*}}\right)^{-3 q(1+w)}+c_{2},
$$

where $c_{1}$ and $c_{2}$ are integration constants. Therefore, using (24) we can express this solution in terms of $T$ as

$$
\begin{aligned}
& F(T)=c_{1} \frac{\tau_{*}^{3 q}}{\sqrt{6} q} \sqrt{-T}+c_{2} \\
& -\frac{(3 q-1)\left(6 q^{2}\right)^{\frac{3 q(1+w)}{2(3 q-1)}} \tau_{*}^{-\frac{3 q(1+w)}{3 q-1}} \rho_{\mathrm{M} *}}{[3 q(2+w)-1]}(-T)^{-\frac{3 q(1+w)}{2(3 q-1)}}
\end{aligned}
$$

Hence, we have reconstructed the $F(T)$ form that generates the power-law scale factor evolution (22). Finally, for completeness we give the expression for the Lagrange multiplier too. In particular, inserting (27) into Eq. (14) we find that

$$
\lambda(\tau)=-2 \rho_{\mathrm{M} *}\left(\frac{\tau}{\tau_{*}}\right)^{-3 q(1+w)}+c_{2}
$$

which using (24) leads to

$$
\lambda(T)=-2 \rho_{\mathrm{M} *}\left(6 q^{2}\right)^{\frac{3 q(1+w)}{2(3 q-1)}} \tau_{*}^{-\frac{3 q(1+w)}{3 q-1}}(-T)^{-\frac{3 q(1+w)}{2(3 q-1)}}+c_{2} .
$$

Lastly, note that in the vacuum case, i.e. when $\rho_{\mathrm{M}}=0$ and $P_{\mathrm{M}}=0$, we find that

$$
F(T)=c_{1} \sqrt{-T}+c_{2},
$$

while

$$
\lambda=c_{2}
$$

Thus, the Lagrange multiplier becomes constant and the $F(T)$ form can be reconstructed without using the expression of the scale factor. However, we mention that in this simple case the theory becomes trivial, since the Lagrangian, in the absence of the constant term, becomes a total derivative [29].

\section{INFLATIONARY COSMOLOGY}

In the previous section we presented unimodular $F(T)$ gravity and we analyzed the procedure with which one can reconstruct the specific $F(T)$ form that can generate a given scale-factor evolution. Hence, in the present section we will apply these in inflationary cosmology, in order to investigate inflation realization in unimodular $F(T)$ gravity. Additionally, we will extract various inflation-related observables, such as the scalar and tensor spectral indices, the running spectral index, and the tensor-to-scalar ratio, and we will compare them with observational data.

\section{A. Slow-roll parameters and inflationary observables}

In every inflationary scenario one needs to calculate the values of various inflation-related observables, such as the scalar spectral index of the curvature perturbations $n_{\mathrm{s}}$, the running $\alpha_{\mathrm{s}} \equiv d n_{\mathrm{s}} / d \ln k$ of the spectral index $n_{\mathrm{s}}$, where $k$ is the absolute value of the wave number $\boldsymbol{k}$, the tensor spectral index $n_{\mathrm{T}}$ and the tensor-to-scalar ratio $r$, since these quantities are determined very accurately by observational data, and thus confrontation can constrain of exclude the scenarios at hand. In principle, the calculation of the above observables requires a detailed and lengthy perturbation analysis. However, one can bypass this procedure by transforming the given scenario to the Einstein frame, where all the inflation information is encoded in the (effective) scalar potential $V(\phi)$, defining the slow-roll parameters $\epsilon, \eta$ and $\xi$ in terms of this potential and its derivatives as [30, 31]

$$
\begin{aligned}
\epsilon & \equiv \frac{M_{p}^{2}}{2}\left(\frac{1}{V} \frac{d V}{d \phi}\right)^{2}, \\
\eta & \equiv \frac{M_{p}^{2}}{V} \frac{d^{2} V}{d \phi^{2}} \\
\xi^{2} & \equiv \frac{M_{p}^{4}}{V^{2}} \frac{d V}{d \phi} \frac{d^{3} V}{d \phi^{3}}
\end{aligned}
$$

(inflation ends when $\epsilon=1$ ), and then using the approximate expressions for the observables in terms of these potential-related slow-roll parameters [31]:

$$
\begin{aligned}
r & \approx 16 \epsilon, \\
n_{\mathrm{s}} & \approx 1-6 \epsilon+2 \eta, \\
\alpha_{\mathrm{s}} & \approx 16 \epsilon \eta-24 \epsilon^{2}-2 \xi^{2}, \\
n_{\mathrm{T}} & \approx-2 \epsilon .
\end{aligned}
$$

However, the above procedure cannot be applied in modified gravity scenarios where the conformal transformation to the Einstein frame is absent, since in this case one cannot define a scalar potential and then the potential-related slow-roll parameters. In such scenarios one should instead introduce the "Hubble slow-roll" parameters $\epsilon_{n}$ (with $n$ positive integer), defined as 31]

$$
\epsilon_{n+1} \equiv \frac{d \ln \left|\epsilon_{n}\right|}{d N}
$$

with $\epsilon_{0} \equiv H_{\text {ini }} / H$ and $N \equiv \ln \left(a / a_{\text {ini }}\right)$ the e-folding number, and where $a_{i n i}$ is the scale factor at the beginning of inflation and $H_{i n i}$ the corresponding Hubble parameter (inflation ends when $\epsilon_{1}=1$ ). In terms of the first three 
$\epsilon_{n}$, which are straightforwardly extracted to be

$$
\begin{aligned}
\epsilon_{1} \equiv & -\frac{\dot{H}}{H^{2}}, \\
\epsilon_{2} \equiv & \frac{\ddot{H}}{H \dot{H}}-\frac{2 \dot{H}}{H^{2}}, \\
\epsilon_{3} \equiv & \left(\ddot{H} H-2 \dot{H}^{2}\right)^{-1} \\
& \cdot\left[\frac{H \dot{H} \ddot{H}-\ddot{H}\left(\dot{H}^{2}+H \ddot{H}\right)}{H \dot{H}}-\frac{2 \dot{H}}{H^{2}}\left(H \ddot{H}-2 \dot{H}^{2}\right)\right],
\end{aligned}
$$

the inflationary observables write as 31]

$$
\begin{aligned}
r & \approx 16 \epsilon_{1}, \\
n_{\mathrm{s}} & \approx 1-2 \epsilon_{1}-2 \epsilon_{2}, \\
\alpha_{\mathrm{s}} & \approx-2 \epsilon_{1} \epsilon_{2}-\epsilon_{2} \epsilon_{3}, \\
n_{\mathrm{T}} & \approx-2 \epsilon_{1} .
\end{aligned}
$$

Obviously, in cases where both the potential-related slowroll parameters and the Hubble slow-roll parameters can be defined, the final expressions for the observables $r, n_{\mathrm{s}}$, $\alpha_{\mathrm{s}}$ and $n_{\mathrm{T}}$ coincide.

In the present work we are interested in investigating inflationary cosmology in the framework of unimodular $F(T)$ gravity. Similarly to usual $F(T)$ gravity, and in contrast with $F(R)$ gravity, in this case there is not a conformal transformation to the Einstein frame [18, 32], where one could find the effective scalar potential and then use it in order to calculate the potential-related slow-roll parameters. Hence, in order to calculate the inflationary observables we must use the Hubble slow-roll parameters (40)-(42), and then insert them into expressions (43)- 46).

Finally, let us stress here that the above procedure pre-assumes that there is only one extra (comparing to general relativity) degree of freedom that drives inflation, and which will affect the scalar and tensor perturbations. However, in general, $F(T)$ gravity possesses three extra degrees of freedom, corresponding to one massive vector field or to one massless vector field and one scalar [33]. Nevertheless, as it was shown in 34], in the case of FLRW geometry, and for viable $F(T)$ scenarios, only one extra degree of freedom arises at the background and linear perturbation levels. Hence, as a first approximation, the observables can be calculated with the aforementioned procedure, through the use of Hubble slow-roll parameters.

\section{B. de Sitter and power-law inflation}

Let us first provide the kinematical expressions for inflation realization. Without loss of generality we focus on two basic inflationary scale-factor evolutions, namely the de Sitter and the power-law ones. The investigation of arbitrary evolutions is straightforward.
For the de Sitter inflation the scale factor has the wellknown exponential form

$$
a(t)=e^{H_{\mathrm{inf}} t},
$$

where $H_{\text {inf }}$ is the constant Hubble parameter at the inflationary stage. In this case, following the procedure of the previous section, and in particular relations (8) and (9), we find

$$
\begin{aligned}
\tau & =\frac{1}{3 H_{\mathrm{inf}}} e^{3 H_{\mathrm{inf}} t}, \\
a(\tau) & =\left(H_{\mathrm{inf}} \tau\right)^{1 / 3} .
\end{aligned}
$$

Similarly, for the power-law inflation the scale factor has the form (20), namely

$$
a(t)=a_{*}\left(\frac{t}{t_{*}}\right)^{p}
$$

where $a_{*}$ is the value of $a$ at time $t_{*}$ and $p$ is a constant. In this case, and as was analyzed in the previous section, in expressions (22) and (23), we obtain

$$
a(\tau)=\left(\frac{\tau}{\tau_{*}}\right)^{q}
$$

with $q=p /(3 p+1)$ and $\tau_{*}=t_{*} /\left[a_{*}^{1 / p}(3 p+1)\right]$. Note that in the limit $p \rightarrow \infty$ and $t_{*} \rightarrow \infty$ with $p / t_{*}=H_{*}=$ const., the power-law expansion (50) gives the de Sitter expansion (47), with $H_{\mathrm{inf}}=3 H_{*}$. Hence, we can study both cases in a simultaneous way, using (51), and thus the unimodular metric (9) becomes

$d s^{2}=\left(\frac{\tau}{\tau_{*}}\right)^{-6 q} d \tau^{2}-\left(\frac{\tau}{\tau_{*}}\right)^{2 q}\left[\left(d x^{1}\right)^{2}+\left(d x^{2}\right)^{2}+\left(d x^{3}\right)^{2}\right]$.

In summary, for $q=1 / 3$ we re-obtain the de Sitter expansion, while for $1 / 4<q<1 / 3$, i.e. for $p>1$, the powerlaw inflation is realized. Additionally, note that for $p<0$, i.e. for $q<0$ or $q>1 / 3$, we acquire $\dot{H}=-p / t^{2}>0$, which corresponds to the realization of super-inflation. Finally, for $0<p \leq 1$, i.e. for $0<q \leq 1 / 4$, accelerated expansion, and thus inflation, is not realized.

Let us now investigate the observables in the case of power-law inflation. As it was shown in 35], it is more convenient to use the e-folding number $N$ as the independent variable (for related considerations, see [36, 37]). Hence, for every function $g$ we have that $\dot{g}=g^{\prime}(N) H(N)$, where primes denote derivatives with respect to $N$. Thus, the Hubble slow-roll parameters (40)-(42) can be reexpressed as

$$
\begin{aligned}
& \epsilon_{1}(N) \equiv-\frac{H^{\prime}(N)}{H(N)}, \\
& \epsilon_{2}(N) \equiv \frac{H^{\prime \prime}(N)}{H^{\prime}(N)}-\frac{H^{\prime}(N)}{H(N)}, \\
& \epsilon_{3}(N) \equiv\left[\frac{H(N) H^{\prime}(N)}{H^{\prime \prime}(N) H(N)-H^{\prime}(N)^{2}}\right] \\
& \cdot\left[\frac{H^{\prime \prime \prime}(N)}{H^{\prime}(N)}-\frac{H^{\prime \prime}(N)^{2}}{H^{\prime}(N)^{2}}-\frac{H^{\prime \prime}(N)}{H(N)}+\frac{H^{\prime}(N)^{2}}{H(N)^{2}}\right] .
\end{aligned}
$$


Let us now consider the power-law inflation of the form (50) or, expressed in unimodular terms, of (51) and (52). For this case we obtain

$$
\begin{aligned}
\epsilon_{1} & =\frac{1}{p}, \\
\epsilon_{2} & =0, \\
\epsilon_{3} & =\frac{1}{p},
\end{aligned}
$$

and thus (43)-(46) give

$$
\begin{aligned}
& r \approx \frac{16}{p}, \\
& n_{\mathrm{s}} \approx 1-\frac{2}{p}, \\
& \alpha_{\mathrm{s}} \approx 0, \\
& n_{\mathrm{T}} \approx-\frac{2}{p},
\end{aligned}
$$

where we remind that $p=q /(1-3 q)$. Finally, eliminating $p$ between (59), (60) we obtain

$$
r=8\left(1-n_{\mathrm{s}}\right) .
$$

Hence, if we take $p=100$, i.e. for $q=0.332$, we acquire $r \approx 0.16 n_{\mathrm{s}} \approx 0.98, \alpha_{\mathrm{s}}=0$, and $n_{\mathrm{T}} \approx-0.02$, which is in good agreement with the Planck results [2]. However, one can see that the power-law inflation is a quite simple scenario and thus the expressions for the observables, although in agreement with observations, are simple and one does not have large parametric freedom to change their values. Hence, in the next subsection we examine a more complicated scenario, with improved phenomenological behavior.

\section{Starobinsky inflation}

Let us consider a simple but efficient model, namely the $R^{2}$ or Starobinsky inflation. In curvature modified gravity, and in particular in $R^{2}$ inflation, the action is given by $S=\int d^{4} x \sqrt{-g}\left\{R+\left[1 /\left(6 M^{2}\right)\right] R^{2}\right\}$, where $M$ is a constant with mass dimension. In such a scenario the Hubble parameter can be described as [38, 39]

$$
H=H_{*}-\frac{M^{2}}{6}\left(t-t_{*}\right),
$$

where $H_{*}$ is the value of the Hubble function at $t=t_{*}$. Straightforwardly, the scale factor reads as $a(t)=a_{*} \exp \left[H_{*}\left(t-t_{*}\right)-\left(M^{2} / 12\right)\left(t-t_{*}\right)^{2}\right]$. For $t \ll t_{*}$, that is at the early stages of inflation, we approximately acquire $a(t)=$ $a_{*} \exp \left\{\left[H_{*}+\left(M^{2} / 6\right) t_{*}\right] t-\left[H_{*}+\left(M^{2} / 12\right) t_{*}\right] t_{*}\right\}$.

Thus, using the expressions extracted in Section III, and in particular (8), in the framework of unimodular gravity and the new time variable we have

$$
\begin{aligned}
& \tau=\bar{\tau}+\frac{a_{*}^{3}}{3 H_{*}+\left(M^{2} / 2\right) t_{*}} \exp \left[\left(3 H_{*}+\frac{M^{2}}{2} t_{*}\right) t\right. \\
& \left.-\left(3 H_{*}+\frac{M^{2}}{4} t_{*}\right) t_{*}\right], \\
& a(\tau)=\left(3 H_{*}+\frac{M^{2}}{2} t_{*}\right)^{1 / 3}(\tau-\bar{\tau})^{1 / 3},
\end{aligned}
$$

where $\bar{\tau}$ is a constant. From (66) we obtain $\mathcal{H}=$ $1 /[3(\tau-\bar{\tau})]$, and therefore, using (13) for the torsion scalar we acquire $T=-6\left[H_{*}+\left(M^{2} / 6\right) t_{*}\right]^{2}$. Thus, we deduce that in the early stage of inflation $T$ is approximately constant.

The e-folding number $N$ at the inflationary stage is defined as

$$
N \equiv \ln \left(\frac{a_{\mathrm{f}}}{a_{\mathrm{i}}}\right)=-\int_{t_{\mathrm{f}}}^{t_{\mathrm{i}}} H(\tilde{t}) d \tilde{t},
$$

where $a_{\mathrm{i}}=a\left(t=t_{\mathrm{i}}\right)$ is the value of the scale factor $a$ at the beginning of inflation $t_{\mathrm{i}}$, and $a_{\mathrm{f}}=a\left(t=t_{\mathrm{f}}\right)$ is its value at the end of inflation $t_{\mathrm{f}}$. Inserting (64) into (67) we acquire

$$
N=-\left(H_{*}+\frac{M^{2}}{6} t_{*}\right)\left(t_{\mathrm{i}}-t_{\mathrm{f}}\right)+\frac{M^{2}}{12}\left(t_{\mathrm{i}}^{2}-t_{\mathrm{f}}^{2}\right),
$$

which for positive $t_{\mathrm{f}}$ can be inverted to express $t_{\mathrm{f}}$ in terms of $N$, namely

$$
\begin{aligned}
t_{\mathrm{f}}=6 & \frac{H_{*}}{M^{2}}+t_{*}+M^{-2}\left\{\left(6 H_{*}+M^{2} t_{*}\right)^{2}\right. \\
& \left.\quad+M^{2}\left\{t_{\mathrm{i}}\left[M^{2}\left(t_{\mathrm{i}}-2 t_{*}\right)-12 H_{*}\right]-12 N\right\}\right\}^{\frac{1}{2}} .
\end{aligned}
$$

We can now use (64) in order to calculate the Hubble slow-roll parameter $\epsilon_{1}$ from (40), namely

$$
\epsilon_{1}=\frac{6 M^{2}}{\left[6 H_{*}-M^{2}\left(t_{\mathrm{f}}-t_{*}\right)\right]^{2}},
$$

and thus eliminating $t_{\mathrm{f}}$ in favor of $N$ using (69) we obtain

$\epsilon_{1}(N)=\frac{6 M^{2}}{\left(6 H_{*}+M^{2} t_{*}\right)^{2}+M^{2}\left\{t_{\mathrm{i}}\left[M^{2}\left(t_{\mathrm{i}}-2 t_{*}\right)-12 H_{*}\right]-12 N\right\}}$.

Similarly, from (41), (42) we obtain

$$
\begin{aligned}
& \epsilon_{2}(N)=2 \epsilon_{1}(N), \\
& \epsilon_{3}(N)=2 \epsilon_{1}(N) .
\end{aligned}
$$

Inserting these into (43)-(46) we find

$$
\begin{aligned}
r(N) & =16 \epsilon_{1}(N), \\
n_{\mathrm{s}}(N) & =1-6 \epsilon_{1}(N), \\
\alpha_{\mathrm{s}}(N) & =-8 \epsilon_{1}^{2}(N), \\
n_{\mathrm{T}}(N) & =-2 \epsilon_{1}(N) .
\end{aligned}
$$


These expressions for the inflationary observables can be in a very good agreement with observations [2]. For instance, taking $t_{\mathrm{i}}=1 / H_{*}, t_{*}=3 / H_{*}, H_{*} / M=0.04$, and for e-folding number $N=50$, we find $r \approx 0.049$ $n_{\mathrm{s}} \approx 0.981, \alpha_{\mathrm{s}}=-7.78 \times 10^{-5}$, and $n_{\mathrm{T}} \approx-0.0062$, while for $N=60$, we find $r \approx 0.053 n_{\mathrm{s}} \approx 0.98$, $\alpha_{\mathrm{s}}=-8.85 \times 10^{-5}$, and $n_{\mathrm{T}} \approx-0.0067$.

We can eliminate the complicated function $\epsilon_{1}(N)$ between (74) and (75), and between (74) and (76), obtaining respectively

$$
r=\frac{8}{3}\left(1-n_{\mathrm{s}}\right)
$$

and

$$
\alpha_{\mathrm{s}}=-\frac{2}{9}\left(1-n_{\mathrm{s}}\right)^{2}
$$

which prove to be very useful, since they allow us to compare the predictions of our scenario with the observational data. In particular, the Planck results [2] suggest that $n_{\mathrm{s}}=0.968 \pm 0.006(68 \% \mathrm{CL}), r<0.11(95 \% \mathrm{CL})$, and $\alpha_{\mathrm{s}}=-0.003 \pm 0.007(68 \% \mathrm{CL})$. The combined analysis of the BICEP2 and Keck Array data with the Planck data shows $r<0.07(95 \% \mathrm{CL})$ [3]. As we can see, using (78) and (79), for $n_{\mathrm{s}} \approx 0.97$ we obtain $r \approx 0.08$ and $\alpha_{\mathrm{s}} \approx-0.0002$, which reveals a very good agreement with observations.

\section{A specific model: $F(T)=-T+\alpha_{1}(-T)^{n}+\Lambda$ and $\lambda(T)=\alpha_{2}+\alpha_{3}(-T)^{m}$}

Let us close the investigation of inflationary cosmology in the framework of unimodular $F(T)$ gravity, by examining a specific model. As we showed in subsection IID, in unimodular $F(T)$ gravity inflation may arise from power-law forms of the $F(T)$ and Lagrange multiplier $\lambda(T)$ functions. Hence, as a specific example we choose

$$
\begin{aligned}
F(T) & =-T+\alpha_{1}(-T)^{n}+\Lambda, \\
\lambda(T) & =\alpha_{2}+\alpha_{3}(-T)^{m}
\end{aligned}
$$

where $\alpha_{1}, \alpha_{2}, \alpha_{3}, \Lambda$ and $n, m$ are constants (the minus sign in front of $T$ is chosen for convenience, since in the usual conventions of $f(T)$ formulation, in FRW geometry $\left.T=-6 H^{2}<0\right)$. In the following we will focus on the case where $n=2, m=1 / 2$. Inserting the above ansatzes into the first Friedmann equation (14), using the relation $d \tau=a^{3}(\tau) d t$, and considering also the matter sector to correspond to radiation, i.e $\rho_{\mathrm{M}}=\rho_{r 0} / a(t)^{4}$, we obtain the following differential equation:

$\Lambda-\alpha_{2}-6 H(t)^{2}-\sqrt{6} \alpha_{3} H(t)+\frac{\rho_{r 0}}{a(t)^{4}}-108 \alpha_{1} H(t)^{4}=0$,

with $H(t) \equiv \dot{a}(t) / a(t)$ and $\rho_{r 0}$ a constant. Choosing small values for $\alpha_{1}$ (such that $\alpha_{1} H(t)^{2} \ll 1$ ) and for $\rho_{r 0}$ (such that $\rho_{r 0} \ll 4 \alpha_{2}-\alpha_{3}^{2}-4 \Lambda$ ) we can extract the solution as

$$
H(t) \approx \frac{\beta_{2}}{\beta_{3}+2 e^{\beta_{1} t}}
$$

where the new constants are defined as

$$
\begin{aligned}
\beta_{1}= & \sqrt{\frac{2}{3}}\left(\alpha_{3}-\sqrt{\alpha_{3}^{2}+4 \Lambda-4 \alpha_{2}}\right) \\
\beta_{2}= & \frac{\rho_{r 0}^{-1}}{2 \sqrt{6}}\left(\sqrt{\alpha_{3}^{2}+4 \Lambda-4 \alpha_{2}}-\alpha_{3}\right) \\
& \cdot \exp \left\{4 \left[\left(\alpha_{3}^{2}+4 \Lambda-4 \alpha_{2}\right)\left(4 \alpha_{2}+\alpha_{3} \sqrt{\alpha_{3}^{2}+4 \Lambda-4 \alpha_{2}}\right)\right.\right. \\
\beta_{3}= & \rho_{r 0}^{-1} \exp \left\{4 \sqrt { \alpha _ { 3 } ^ { 2 } + 4 \Lambda - 4 \alpha _ { 2 } } \left[\alpha_{3}\left(1-4 \alpha^{2}+4 \Lambda\right)\right.\right. \\
& \left.\left.+\alpha_{3}^{2}+\left(4 \alpha_{2}-1\right) \sqrt{\alpha_{3}^{2}+4 \Lambda-4 \alpha_{2}}\right] C_{1}\right\},(84)
\end{aligned}
$$

with $C_{1}$ is an integration constant.

Let us now use the solution (83) in order to calculate the inflationary observables. We start by calculating the e-folding number $N$ defined in (67), obtaining

$$
N=\frac{\beta_{2}}{\beta_{3} \beta_{1}}\left[\beta_{1}\left(t_{i}-t_{f}\right)+\ln \left(\frac{\beta_{3}+2 e^{\beta_{1} t_{f}}}{\beta_{3}+2 e^{\beta_{1} t_{i}}}\right)\right]
$$

where $t_{\mathrm{i}}$ is the beginning of inflation and $t_{\mathrm{f}}$ its end. Relation (85) can be easily inverted to give $t_{i}(N)$. Next, we use (83) in oder to calculate the Hubble slow-roll parameters from (40), (41), (42), acquiring

$$
\begin{aligned}
\epsilon_{1} & =\frac{2 \beta_{1} e^{\beta_{1} t_{i}}}{\beta_{2}} \\
\epsilon_{2} & =\frac{\beta_{1}\left(\beta_{3}+2 e^{\beta_{1} t_{i}}\right)}{\beta_{2}} \\
\epsilon_{3} & =\frac{2 \beta_{1} e^{\beta_{1} t_{i}}}{\beta_{2}}
\end{aligned}
$$

Inserting these into (43)-46) we find

$$
\begin{aligned}
& r=\frac{32 \beta_{1} e^{\beta_{1} t_{i}}}{\beta_{2}}, \\
& n_{\mathrm{s}}=1-\frac{2 \beta_{1}\left(\beta_{3}+4 e^{\beta_{1} t_{i}}\right)}{\beta_{2}}, \\
& \alpha_{\mathrm{s}}=-\frac{8 \beta_{1}^{2} e^{\beta_{1} t_{i}}\left(\beta_{3}+2 e^{\beta_{1} t_{i}}\right)}{\beta_{2}^{2}}, \\
& n_{\mathrm{T}}=-\frac{4 \beta_{1} e^{\beta_{1} t_{i}}}{\beta_{2}} .
\end{aligned}
$$

Hence, we can insert 855) into the above relations in 
order to eliminate $t_{i}$ in favor of $N$, obtaining

$$
\begin{aligned}
& r(N)=\frac{32 \beta_{3} \beta_{1} e^{\beta_{1}\left(\frac{\beta_{3}}{\beta_{2}} N+t_{f}\right)}}{\beta_{2}\left[\beta_{3}-2\left(e^{\frac{\beta_{1} \beta_{3}}{\beta_{2}} N}-1\right) e^{\beta_{1} t_{f}}\right]}, \\
& n_{\mathrm{S}}(N)=1-\frac{2 \beta_{3} \beta_{1}\left[\beta_{3}+2 e^{\beta_{1} t_{f}}\left(1+e^{\frac{\beta_{1} \beta_{3}}{\beta_{2}} N}\right)\right]}{\beta_{2}\left[\beta_{3}-2\left(e^{\frac{\beta_{1} \beta_{3}}{\beta_{2}} N}-1\right) e^{\beta_{1} t_{f}}\right]}, \\
& \alpha_{\mathrm{S}}(N)=-\frac{\left.8 \beta_{3}^{2} \beta_{1}^{2}\left(\beta_{3}+2 e^{\beta_{1} t_{f}}\right) e^{\beta_{1}\left(\frac{\beta_{3}}{\beta_{2}} N+t_{f}\right.}\right)}{\beta_{2}^{2}\left[\beta_{3}-2\left(e^{\frac{\beta_{1} \beta_{3}}{\beta_{2}} N}-1\right) e^{\beta_{1} t_{f}}\right]}, \\
& n_{\mathrm{T}}(N)=-\frac{4 \beta_{3} \beta_{1} e^{\beta_{1}\left(\frac{\beta_{3}}{\beta_{2}} N+t_{f}\right)}}{\beta_{2}\left[\beta_{3}-2\left(e^{\frac{\beta_{1} \beta_{3}}{\beta_{2}} N}-1\right) e^{\beta_{1} t_{f}}\right]} .
\end{aligned}
$$

Finally, we can insert (94) into 93 and (95), in order to eliminate $N$ and $t_{f}$ in favor of $n_{\mathrm{s}}$, resulting to

$$
\begin{aligned}
& r=4\left(1-n_{\mathrm{s}}\right)-\frac{8 \beta_{3} \beta_{1}}{\beta_{2}}, \\
& \alpha_{\mathrm{s}}=-\frac{1}{4}\left(n_{\mathrm{s}}-1\right)^{2}+\frac{\beta_{3}^{2} \beta_{1}^{2}}{\beta_{2}^{2}}, \\
& n_{\mathrm{T}}=-\frac{1}{2}\left(1-n_{\mathrm{s}}\right)+\frac{\beta_{3} \beta_{1}}{\beta_{2}},
\end{aligned}
$$

or, restoring the original parameters using (84), we obtain

$$
\begin{aligned}
& r=4\left(1-n_{\mathrm{s}}\right) \\
& +32 \exp \left\{\frac{4\left[\alpha_{3}^{3}+4 \alpha_{3} \Lambda+4 \alpha_{2}\left(\sqrt{\alpha_{3}^{2}+4 \Lambda-4 \alpha_{2}}-\alpha_{3}\right)\right] C_{1}}{\sqrt{\alpha_{3}^{2}+4 \Lambda-4 \alpha_{2}}}\right\},( \\
& \alpha_{\mathrm{s}}=-\frac{1}{4}\left(n_{\mathrm{s}}-1\right)^{2} \\
& +16 \exp \left\{\frac{4\left[\alpha_{3}^{3}+4 \alpha_{3} \Lambda+4 \alpha_{2}\left(\sqrt{\alpha_{3}^{2}+4 \Lambda-4 \alpha_{2}}-\alpha_{3}\right)\right] C_{1}}{\sqrt{\alpha_{3}^{2}+4 \Lambda-4 \alpha_{2}}}\right\},( \\
& n_{\mathrm{T}}=-\frac{1}{2}\left(1-n_{\mathrm{s}}\right) \\
& -4 \exp \left\{\frac{4\left[\alpha_{3}^{3}+4 \alpha_{3} \Lambda+4 \alpha_{2}\left(\sqrt{\alpha_{3}^{2}+4 \Lambda-4 \alpha_{2}}-\alpha_{3}\right)\right] C_{1}}{\sqrt{\alpha_{3}^{2}+4 \Lambda-4 \alpha_{2}}}\right\} .
\end{aligned}
$$

Relations (100)-1102) prove to be very useful, since they allow us to compare the predictions of our scenario with the observational data. In particular, in Fig. 11 we use (100) and we present the estimated tensor-to-scalar ratio of the specific scenario (80)-81) of inflation in unimodular $F(T)$ gravity, for two cases, on top of the $1 \sigma$ and $2 \sigma$ contours of the Planck 2013 results [40] as well as of the Planck 2015 results [41]. Additionally, in Fig. 2 we use (101) and we show the predictions of our scenario for the running spectral index $\alpha_{\mathrm{s}}$ on top of the $1 \sigma$ and $2 \sigma$ contours of the Planck 2013 results [40] as well as of the Planck 2015 results 41]. The agreement with observations is inside the $2 \sigma$ region for the tensor-to-scalar ratio and it is very satisfactory for the running spectral index. We mention that the agreement with observations is obtained through the unimodular construction, since taking $\lambda$ to zero, i.e. for $\alpha_{2}, \alpha_{3} \rightarrow 0$, one arrives to unacceptable deviations.

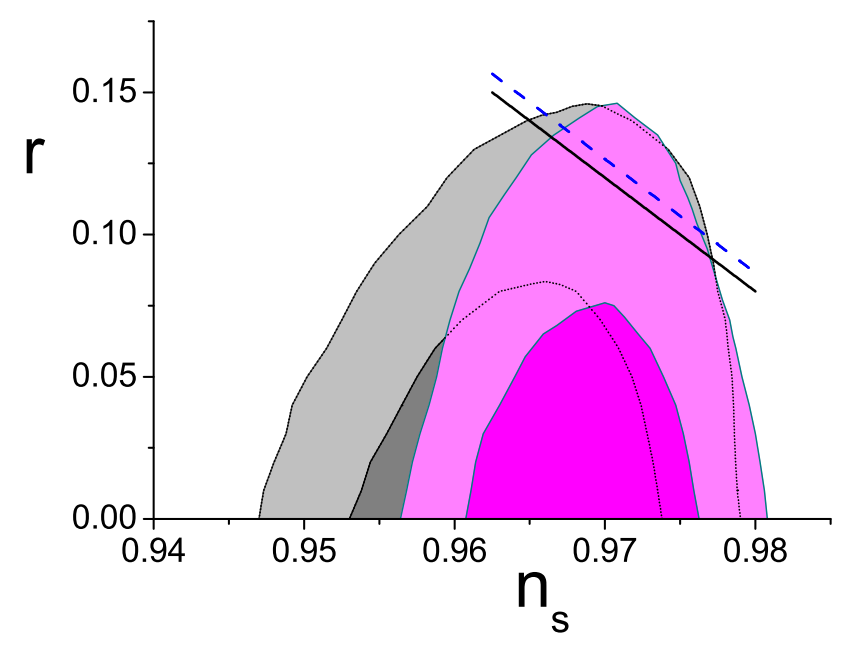

Figure 1: $1 \sigma$ (magenta) and $2 \sigma$ (light magenta) contours for Planck 2015 results $\left(T T+\right.$ low $P+$ lensing $\left.+B A O+J L A+H_{0}\right)$ [41], and $1 \sigma$ (grey) and $2 \sigma$ (light grey) contours for Planck 2013 results (Planck $+W P+B A O$ ) [40] (note that the $1 \sigma$ region of Planck 2013 results is behind the Planck 2015 results, hence we mark its boundary by a dotted curve), on $n_{\mathrm{s}}-r$ plane. Additionally, we present the predictions of the specific scenario (80)- 81) of inflation in unimodular $F(T)$ gravity, with $n=2, m=1 / 2$, and for $\alpha_{2}=0.5, \alpha_{3}=2, \Lambda=0.1$, $C_{1}=-1$ (black - solid curve), and for $\alpha_{2}=1, \alpha_{3}=0.5, \Lambda=$ $1, C_{1}=-0.5$ (blue - dashed curve), in units where $2 \kappa^{2}=1$.

\section{GRACEFUL EXIT FROM THE INFLATIONARY STAGE}

In this section we are interested in investigating the graceful exit from inflation in unimodular $F(T)$ gravity. In general, if the de Sitter solution describing the inflationary stage is unstable, then the graceful exit from inflation can be realized. When the de Sitter inflation occurs the Hubble parameter is described by $H=H_{\mathrm{inf}}$, where $H_{\text {inf }}$ is a positive constant. In order to examine the instability of the de Sitter solution, in general one considers the perturbations of the Hubble parameter, which can be expressed as

$$
\begin{aligned}
H & =H_{\mathrm{inf}}+H_{\mathrm{inf}} \delta(t), \\
\delta(t) & \equiv \delta_{0} e^{\beta t}
\end{aligned}
$$

with $\delta_{0}$ and $\beta$ constants. In (103) the term $H_{\text {inf }} \delta(t)$ describes the perturbations from the de Sitter solution, i.e. the constant part $H_{\text {inf }}$ of the Hubble parameter, and thus 


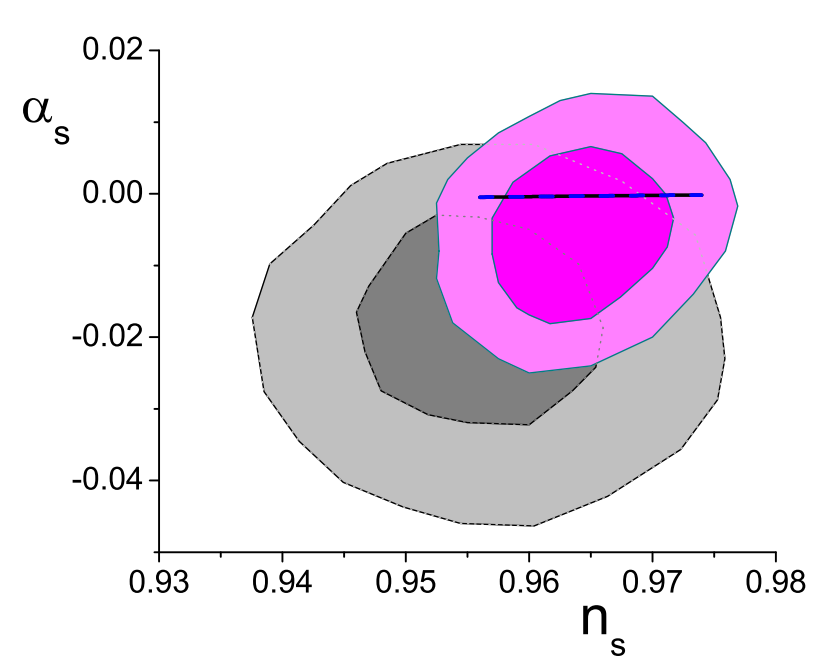

Figure 2: $1 \sigma$ (magenta) and $2 \sigma$ (light magenta) contours for Planck 2015 results (TT, TE, EE + lowP) [41], and $1 \sigma$ (grey) and $2 \sigma$ (light grey) contours for Planck 2013 results $\left(\Lambda C D M+\right.$ running + tensors) [40], on $n_{\mathrm{s}}-\alpha_{\mathrm{s}}$ plane. Additionally, we present the predictions of the specific scenario (80)- 81) of inflation in unimodular $F(T)$ gravity, with $n=2$, $m=1 / 2$, and for $\alpha_{2}=0.5, \alpha_{3}=2, \Lambda=0.1, C_{1}=-1$ (black - solid curve), and for $\alpha_{2}=1, \alpha_{3}=0.5, \Lambda=1, C_{1}=-0.5$ (blue - dashed curve), in units where $2 \kappa^{2}=1$. The two curves are indistinguishable in the resolution scale of the figure.

we assume $|\delta(t)| \ll 1$. Note that in this way we can quantify the instability of the de Sitter solution. If we obtain a positive solution of $\beta$ then the value of $|\delta(t)|$ increases in time during inflation, and thus the de Sitter solution is unstable. Consequently, the inflationary stage ends successfully and the universe can enter the reheating stage and its standard thermal history.

Let us focus on the specific scenario analyzed in subsection IIID, In this case, the solution for the Hubble function (83), in the $|\delta(t)| \ll 1$ regime, can take the form (103) with $H_{\text {inf }}=\beta_{2} / \beta_{3}, \delta_{0}=-2 / \beta_{3}$ and $\beta=\beta_{1}$. Hence, one can easily deduce that for $\beta_{1}>0$ we have a graceful exit from the inflationary regime.

We close this section by making some comments on the difference between the present scenario of inflation in unimodular $F(T)$ gravity and $R^{2}$ inflation (Starobinsky inflation), focusing on the graceful exit. In particular, in $R^{2}$ inflation, for the flat FLRW geometry, by differentiating an equation derived from the gravitational field equations in the absence of matter 10], in terms of $t$, one finds

$$
\dddot{R}+3 \dot{H} \dot{R}+3 H \ddot{R}+M^{2} \dot{R}=0,
$$

where $R=6\left(H^{2}+\dot{H}\right)$. Inserting (103), with $\delta(t)=e^{\tilde{\beta} t}$, into Eq. 105 and keeping first-order terms in $\delta(t)$, we eventually acquire

$$
\tilde{\beta}\left[\tilde{\beta}^{3}+10 H_{\mathrm{inf}} \tilde{\beta}^{2}+\left(24 H_{\mathrm{inf}}^{2}+M^{2}\right) \tilde{\beta}+4 H_{\mathrm{inf}} M^{2}\right]=0 .
$$

It is seen from Eq. (106) that even if there exists a real solution for $\tilde{\beta}$, it would be a non-positive solution, which implies that through the perturbative analysis the instability of the de Sitter solution does not appear. In summary, the de Sitter solution for $R^{2}$ inflation would be more stable than that for inflation in unimodular $F(T)$ gravity. This difference between $R^{2}$ inflation and inflation in unimodular $F(T)$ gravity acts as an advantage for the latter.

\section{CONCLUSIONS}

In the present paper, we have investigated the inflationary realization in the context of unimodular $F(T)$ gravity. The action of the theory is based on the $F(T)$ modification of teleparallel gravity, in which one imposes the unimodular condition through the use of Lagrange multipliers. Hence, we have developed the general reconstruction procedure of the $F(T)$ form that can give rise to a given scale-factor evolution.

Having presented the general machinery, we have applied it in the inflationary regime. In particular, we have extracted the Hubble slow-roll parameters that allow us to calculate various inflation-related observables, such as the scalar spectral index and its running, the tensor-toscalar ratio, and the tensor spectral index. Then, we examined the particular cases of de Sitter and powerlaw inflation, of Starobinsky inflation, as well as inflation in a specific model of unimodular $F(T)$ gravity. As we showed, in all cases the predictions of our scenarios are in a very good agreement with observational data from Planck probe.

Apart from the very satisfactory agreement with observations, the scenario of inflation in unimodular $F(T)$ gravity has the additional advantage that it always allows for a graceful exit for specific regions of the model parameters, as it can be seen by examining the instability of the de Sitter phase. This is in contrast with inflationary realizations in curvature-based modified gravity, such as the Starobinsky inflation, where a graceful exit is not guaranteed. The above features make inflation in unimodular $F(T)$ gravity a successful candidate for the description of the early universe.

\section{Acknowledgments}

This work was partially supported by the JSPS Grantin-Aid for Young Scientists (B) \# 25800136 and the research-funds provided by Fukushima University (K.B.), and MINECO (Spain) project FIS2013-44881 (S.D.O.). 
[1] S. Perlmutter et al. [Supernova Cosmology Project Collaboration], Astrophys. J. 517, 565 (1999). A. G. Riess et al. [Supernova Search Team Collaboration], Astron. J. 116, 1009 (1998).

[2] P. A. R. Ade et al. [Planck Collaboration], arXiv:1502.01589 [astro-ph.CO]; P. A. R. Ade et al. [Planck Collaboration], arXiv:1502.02114 [astro-ph.CO].

[3] P. A. R. Ade et al. [BICEP2 and Keck Array Collaborations], Phys. Rev. Lett. 116, 031302 (2016).

[4] G. Hinshaw et al. [WMAP Collaboration], Astrophys. J. Suppl. 208, 19 (2013).

[5] M. Tegmark et al. [SDSS Collaboration], Phys. Rev. D 69, 103501 (2004).

[6] B. Jain and A. Taylor, Phys. Rev. Lett. 91, 141302 (2003).

[7] K. Sato, Mon. Not. Roy. Astron. Soc. 195, 467 (1981); A. H. Guth, Phys. Rev. D 23, 347 (1981); A. D. Linde, Phys. Lett. B 108, 389 (1982); A. Albrecht and P. J. Steinhardt, Phys. Rev. Lett. 48, 1220 (1982).

[8] P. J. E. Peebles and B. Ratra, Rev. Mod. Phys. 75, 559 (2003).

[9] Y. F. Cai, E. N. Saridakis, M. R. Setare and J. Q. Xia, Phys. Rept. 493, 1 (2010); K. Bamba, S. Capozziello, S. Nojiri and S. D. Odintsov, Astrophys. Space Sci. 342, 155 (2012).

[10] S. Nojiri and S. D. Odintsov, Phys. Rept. 505, 59 (2011); S. Nojiri and S. D. Odintsov, eConf C 0602061 (2006) 06 [Int. J. Geom. Meth. Mod. Phys. 4, 115 (2007)]; S. Capozziello and M. De Laurentis, Phys. Rept. 509, 167 (2011); A. de la Cruz-Dombriz and D. Sáez-Gómez, Entropy 14, 1717 (2012); A. Joyce, B. Jain, J. Khoury and M. Trodden, Phys. Rept. 568, 1 (2015); K. Koyama, Rept. Prog. Phys. 79, 046902 (2016); K. Bamba and S. D. Odintsov, Symmetry 7, 1, 220 (2015).

[11] H. A. Buchdahl, Mon. Not. Roy. Astron. Soc. 150, 1 (1970); S. Capozziello, S. Carloni and A. Troisi, Recent Res. Dev. Astron. Astrophys. 1, 625 (2003); S. Nojiri and S. D. Odintsov, Phys. Rev. D 68, 123512 (2003); S. M. Carroll, V. Duvvuri, M. Trodden and M. S. Turner, Phys. Rev. D 70, 043528 (2004).

[12] A. Einstein 1928, Sitz. Preuss. Akad. Wiss. p. 217; ibid p. 224 ;

[13] K. Hayashi and T. Shirafuji, Phys. Rev. D 19, 3524 (1979); Addendum-ibid. 24, 3312 (1982).

[14] R. Aldrovandi, J.G. Pereira, Teleparallel Gravity: An Introduction, Springer, Dordrecht, 2013.

[15] J. W. Maluf, Annalen Phys. 525, 339 (2013).

[16] G. R. Bengochea and R. Ferraro, Phys. Rev. D 79, 124019 (2009).

[17] E. V. Linder, Phys. Rev. D 81, 127301 (2010).

[18] Y. F. Cai, S. Capozziello, M. De Laurentis and E. N. Saridakis, Rept. Prog. Phys. 79, no. 10, 106901 (2016).

[19] R. Ferraro and F. Fiorini, Phys. Rev. D 75, 084031 (2007); R. Ferraro and F. Fiorini, Phys. Rev. D 78, 124019 (2008).

[20] P. Wu, H. W. Yu, Phys. Lett. B693, 415 (2010); R. J. Yang, Eur. Phys. J. C 71, 1797 (2011); G. R. Bengochea, Phys. Lett. B695, 405 (2011); P. Wu and H. W. Yu, Eur. Phys. J. C 71, 1552 (2011); K. Bamba, C. Q. Geng and C. C. Lee, arXiv:1008.4036 [astroph.CO]; J. B. Dent, S. Dutta, E. N. Saridakis, JCAP
1101, 009 (2011); K. Karami and A. Abdolmaleki, Res. Astron. Astrophys. 13, 757 (2013); R. Zheng and Q. G. Huang, JCAP 1103, 002 (2011); K. Bamba, C. Q. Geng, C. C. Lee and L. W. Luo, JCAP 1101, 021 (2011); Y. Zhang, H. Li, Y. Gong and Z. H. Zhu, JCAP 1107, 015 (2011); Y. -F. Cai, S. -H. Chen, J. B. Dent, S. Dutta, E. N. Saridakis, Class. Quant. Grav. 28, 215011 (2011); M. Sharif, S. Rani, Mod. Phys. Lett. A26, 1657 (2011); S. Chattopadhyay and U. Debnath, Int. J. Mod. Phys. D 20, 1135 (2011); S. Capozziello, V. F. Cardone, H. Farajollahi and A. Ravanpak, Phys. Rev. D 84, 043527 (2011); X. h. Meng and Y. b. Wang, Eur. Phys. J. C 71, 1755 (2011); M. H. Daouda, M. E. Rodrigues and M. J. S. Houndjo, Eur. Phys. J. C 72, 1890 (2012).

[21] C. Q. Geng, C. C. Lee, E. N. Saridakis and Y. P. Wu, Phys. Lett. B 704, 384 (2011); K. Bamba and C. Q. Geng, JCAP 1111, 008 (2011); Y. P. Wu and C. Q. Geng, Phys. Rev. D 86, 104058 (2012); C. Q. Geng, C. C. Lee and E. N. Saridakis, JCAP 1201, 002 (2012); K. Bamba, R. Myrzakulov, S. Nojiri and S. D. Odintsov, Phys. Rev. D 85, 104036 (2012); H. Wei, X. J. Guo and L. F. Wang, Phys. Lett. B 707, 298 (2012); K. Atazadeh and F. Darabi, Eur.Phys.J. C72 (2012) 2016; H. Farajollahi, A. Ravanpak and P. Wu, Astrophys. Space Sci. 338, 23 (2012); K. Karami and A. Abdolmaleki, JCAP 1204 (2012) 007; V. F. Cardone, N. Radicella and S. Camera, Phys. Rev. D 85, 124007 (2012); M. Jamil, D. Momeni and R. Myrzakulov, Eur. Phys. J. C 72, 2267 (2012); Y. C. Ong, K. Izumi, J. M. Nester and P. Chen, Phys. Rev. D 88 (2013) 2, 024019; J. Amoros, J. de Haro and S. D. Odintsov, Phys. Rev. D 87, 104037 (2013); R. X. Miao, M. Li and Y. G. Miao, JCAP 1111, 033 (2011); K. Bamba, S. D. Odintsov and D. Sáez-Gómez, Phys. Rev. D 88, 084042 (2013); S. Nesseris, S. Basilakos, E. N. Saridakis and L. Perivolaropoulos, Phys. Rev. D 88, 103010 (2013); K. Bamba, S. Capozziello, M. De Laurentis, S. 'i. Nojiri and D. Sáez-Gómez, Phys. Lett. B 727, 194 (2013); G. Kofinas and E. N. Saridakis, Phys. Rev. D 90, 084044 (2014); T. Harko, F. S. N. Lobo, G. Otalora and E. N. Saridakis, Phys. Rev. D 89, 124036 (2014); J. Haro and J. Amoros, JCAP 1412 (2014) 12, 031; C. Q. Geng, C. Lai, L. W. Luo and H. H. Tseng, Phys. Lett. B 737, 248 (2014); W. El Hanafy and G. G. L. Nashed, Eur. Phys. J. C 75, 279 (2015); F. Darabi, M. Mousavi and K. Atazadeh, Phys. Rev. D 91, 084023 (2015); S. Capozziello, O. Luongo and E. N. Saridakis, Phys. Rev. D 91 (2015) 12, 124037; K. Bamba, arXiv:1504.04457 [gr-qc]; G. L. Nashed, Gen. Rel. Grav. 47 (2015) 7, 75; S. Bahamonde, C. G. Böhmer and M. Wright, Phys. Rev. D 92, 104042 (2015).

[22] J. L. Anderson and D. Finkelstein, Am. J. Phys. 39, 901 (1971); W. Buchmuller and N. Dragon, Phys. Lett. B 207, 292 (1988); M. Henneaux and C. Teitelboim, Phys. Lett. B 222, 195 (1989); W. G. Unruh, Phys. Rev. D 40, 1048 (1989); Y. J. Ng and H. van Dam, J. Math. Phys. 32, 1337 (1991); D. R. Finkelstein, A. A. Galiautdinov and J. E. Baugh, J. Math. Phys. 42, 340 (2001); E. Alvarez, JHEP 0503, 002 (2005); E. Alvarez, D. Blas, J. Garriga and E. Verdaguer, Nucl. Phys. B 756, 148 (2006); A. H. Abbassi and A. M. Abbassi, Class. Quant. Grav. 25, 175018 (2008); G. F. R. El- 
lis, H. van Elst, J. Murugan and J. P. Uzan, Class. Quant. Grav. 28, 225007 (2011); P. Jain, Mod. Phys. Lett. A 27, 1250201 (2012); N. K. Singh, Mod. Phys. Lett. A 28, 1350130 (2013); C. Barceló, R. CarballoRubio and L. J. Garay, Phys. Rev. D 89, 124019 (2014); C. Gao, R. H. Brandenberger, Y. Cai and P. Chen, JCAP 1409, 021 (2014); C. Barceló, R. Carballo-Rubio and L. J. Garay, arXiv:1406.7713 [gr-qc]; A. Padilla and I. D. Saltas, Eur. Phys. J. C 75, 561 (2015); I. D. Saltas, Phys. Rev. D 90, 124052 (2014); J. Kluson, Phys. Rev. D 91, 064058 (2015); A. Eichhorn, JHEP 1504, 096 (2015);

E. Álvarez, S. González-Martín, M. Herrero-Valea and C. P. Martín, JHEP 1508, 078 (2015); A. Basak, O. Fabre and S. Shankaranarayanan, arXiv:1511.01805 [gr-qc]; D. J. Burger, G. F. R. Ellis, J. Murugan and A. Weltman, arXiv:1511.08517 [hep-th].

[23] S. Weinberg, Rev. Mod. Phys. 61, 1 (1989);

P. J. E. Peebles and B. Ratra, Rev. Mod. Phys. 75, 559 (2003).

[24] I. Cho and N. K. Singh, Class. Quant. Grav. 32, 135020 (2015).

[25] P. Jain, P. Karmakar, S. Mitra, S. Panda and N. K. Singh, JCAP 1205, 020 (2012); P. Jain, A. Jaiswal, P. Karmakar, G. Kashyap and N. K. Singh, JCAP 1211, 003 (2012);

[26] S. Nojiri, S. D. Odintsov and V. K. Oikonomou, arXiv:1512.07223 [gr-qc]; S. Nojiri, S. D. Odintsov and V. K. Oikonomou, arXiv:1601.04112 [gr-qc]; D. SáezGómez, arXiv:1602.04771 [gr-qc].

[27] S. B. Nassur, C. Ainamon, M. J. S. Houndjo and J. Tossa, arXiv:1602.03172 [gr-qc].

[28] E. A. Lim, I. Sawicki and A. Vikman, JCAP 1005, 012 (2010); S. Capozziello, J. Matsumoto, S. Nojiri and S. D. Odintsov, Phys. Lett. B 693, 198 (2010); S. Capozziello, A. N. Makarenko and S. D. Odintsov, Phys. Rev. D 87, 084037 (2013);
[29] S. Basilakos, S. Capozziello, M. De Laurentis, A. Paliathanasis and M. Tsamparlis, Phys. Rev. D 88, 103526 (2013); A. Paliathanasis, S. Basilakos, E. N. Saridakis, S. Capozziello, K. Atazadeh, F. Darabi and M. Tsamparlis, Phys. Rev. D 89, 104042 (2014).

[30] J. E. Lidsey, A. R. Liddle, E. W. Kolb, E. J. Copeland, T. Barreiro and M. Abney, Rev. Mod. Phys. 69, 373 (1997); D. H. Lyth and A. Riotto, Phys. Rept. 314, 1 (1999); D. S. Gorbunov and V. A. Rubakov, Introduction to the theory of the early universe: Cosmological perturbations and inflationary theory (Hackensack, USA: World Scientific, 2011).

[31] J. Martin, C. Ringeval and V. Vennin, Phys. Dark Univ. 5-6, 75 (2014).

[32] R. J. Yang, Europhys. Lett. 93, 60001 (2011).

[33] M. Li, R. X. Miao and Y. G. Miao, JHEP 1107, 108 (2011).

[34] B. Li, T. P. Sotiriou and J. D. Barrow, Phys. Rev. D 83, 104017 (2011); S. H. Chen, J. B. Dent, S. Dutta and E. N. Saridakis, Phys. Rev. D 83, 023508 (2011).

[35] K. Bamba, S. Nojiri and S. D. Odintsov, Phys. Lett. B 737, 374 (2014).

[36] K. Bamba, S. Nojiri, S. D. Odintsov and D. Sáez-Gómez, Phys. Rev. D 90, 124061 (2014).

[37] V. Mukhanov, Fortsch. Phys. 63, 36 (2015).

[38] A. A. Starobinsky, Phys. Lett. B 91, 99 (1980).

[39] J. D. Barrow and S. Cotsakis, Phys. Lett. B 214, 515 (1988); S. D. Odintsov and V. K. Oikonomou, Phys. Rev. D 92, 024016 (2015); S. D. Odintsov and V. K. Oikonomou, Phys. Rev. D 92, 124024 (2015);

[40] P. A. R. Ade et al. [Planck Collaboration], Astron. Astrophys. 571, A22 (2014); arXiv:1303.5082 [astro-ph.CO]].

[41] P. A. R. Ade et al. [Planck Collaboration], arXiv:1502.01589 [astro-ph.CO]. 